\title{
Extended Weyl Calculus and Application to the Phase-Space Schrödinger Equation
}

\author{
Maurice A. de Gosson \\ Universität Potsdam, Inst. f. Mathematik \\ Am Neuen Palais 10, Pf 601533 \\ D-14415 Potsdam (Germany) \\ E-mail: maurice.degosson@gmail.com
}

\begin{abstract}
We show that the Schrödinger equation in phase space proposed by Torres-Vega and Frederick is canonical in the sense that it is a natural consequence of the extended Weyl calculus obtained by letting the Heisenberg group act on functions (or half-densities) defined on phase space. This allows us, in passing, to solve rigorously the TF equation for all quadratic Hamiltonians.
\end{abstract}

\section{Introduction}

The theory of phase-space Schrödinger equation

$$
i \hbar \frac{\partial}{\partial t} \Psi(x, p, t)=H\left(x+i \hbar \partial_{p},-i \hbar \partial_{x}\right) \Psi(x, p, t)
$$

corresponding to the quantization rules

$$
x_{j} \longmapsto x_{j}+i \hbar \frac{\partial}{\partial p_{j}}, p_{j} \longmapsto-i \hbar \frac{\partial}{\partial x_{j}}
$$

proposed by Torres-Vega and Frederick [14, 15, 12, (TF) has deservedly received much attention these last years (see for instance [1, 7, 9, 10, 12, 16]). TF arrive at their equation by using a generalized version of the Husimi transform in which coherent states are used as pseudo-transition matrix elements via a certain kernel $K_{c s}$. (We take the opportunity to point out that this kernel has been extensively studied in the mathematical literature under the names of "wavepacket transform" or "FBI transform"; see e.g. 13]). We intend to show that TF's choice corresponds to a natural extension of Weyl calculus, and is therefore in a sense canonical. 


\section{Notations}

We will use the collective notations $x=\left(x_{1}, \ldots, x_{n}\right), p=\left(p_{1}, \ldots, p_{n}\right)$, and $z=$ $(x, p)$. We denote by $\sigma$ the canonical symplectic form on the phase space $\mathbb{R}_{z}^{2 n}=$ $\mathbb{R}_{x}^{n} \times \mathbb{R}_{p}^{n}$ :

$$
\sigma\left(z, z^{\prime}\right)=\left(z^{\prime}\right)^{T} J z \quad, \quad J=\left[\begin{array}{cc}
0 & I \\
-I & 0
\end{array}\right]
$$

if $z=(x, p), z^{\prime}=\left(x^{\prime} p^{\prime}\right)$. We denote by $S p(n)$ the real symplectic group; it consists of all linear automorphisms $S$ of $\mathbb{R}_{z}^{2 n}$ such that $S^{T} J S=S J S^{T}=J$. $\mathcal{S}\left(\mathbb{R}^{n}\right)$ is the Schwartz space of rapidly decreasing functions on $\mathbb{R}^{n}$ and its dual $\mathcal{S}^{\prime}\left(\mathbb{R}^{n}\right)$ the space of tempered distributions. The scalar product of two $n$-vectors $x$ and $p$ is denoted by $x p$, and we will write $\partial_{z}=\left(\partial_{x}, \partial_{p}\right)$ where

$$
\partial_{x}=\left(\frac{\partial}{\partial x_{1}}, \cdots, \frac{\partial}{\partial x_{n}}\right), \partial_{p}=\left(\frac{\partial}{\partial p_{1}}, \cdots, \frac{\partial}{\partial p_{n}}\right) .
$$

\section{Heisenberg-Weyl Operators on Phase Space}

Let us recall the definition of the Heisenberg-Weyl operators from the "Schrödingerian" point of view (see e.g. [8] ). The flow $\left(f_{t}\right)$ determined by the Hamiltonian $H_{z_{0}}(z)=\sigma\left(z, z_{0}\right)$ is given by $f_{t}(z)=z+t z_{0}$ hence the time-one mapping $f_{1}$ is just the phase-space translation $T\left(z_{0}\right): z \longmapsto z+z_{0}$; the action of that translation on functions $\Psi=\Psi(z)$ is defined by "push-forward";

$$
T\left(z_{0}\right) \Psi(z)=\Psi\left(z-z_{0}\right) .
$$

The quantized version of the Hamiltonian $H_{z_{0}}$ is the operator $\widehat{H}_{z_{0}}=\sigma\left(\widehat{z}, z_{0}\right)$ with $\widehat{z}=\left(x,-i \hbar \partial_{x}\right)$, and the solution of the corresponding Schrödinger equation

$$
i \hbar \frac{\partial \psi}{\partial t}=\sigma\left(\widehat{z}, z_{0}\right) \psi \quad, \quad \psi(x, 0)=\psi_{0}(x)
$$

is the function

$$
\psi(x, t)=\exp \left[\frac{i}{\hbar}\left(t p_{0} x-\frac{t^{2}}{2} p_{0} x_{0}\right)\right] \psi_{0}\left(x-t x_{0}\right) .
$$

The value of $\psi$ a point $x$ at time $t=1$ is denoted by $\widehat{T}\left(z_{0}\right) \psi(x)$ :

$$
\widehat{T}\left(z_{0}\right) \psi(x)=e^{\frac{i}{\hbar}\left(p_{0} x-\frac{1}{2} p_{0} x_{0}\right)} \psi\left(x-x_{0}\right) ;
$$

$\widehat{T}\left(z_{0}\right)$ is by definition the Heisenberg-Weyl operator associated to the translation $T\left(z_{0}\right)$ (see 8 ). So much for the traditional point of view. Let us shortly review the notion of phase as studied in our paper [5]. Consider a Lagrangian manifold $\mathbb{V}_{0}^{n}$ in $\mathbb{R}_{z}^{2 n}$; we assume for simplicity that $\mathbb{V}_{0}^{n}$ is simply connected (this restriction, which can be alleviated by passing to the universal covering, is of no consequence 
for the argument that follows). Choosing, once for all, a base point $\bar{z}$ in $\mathbb{V}_{0}^{n}$ we define the phase of $\mathbb{V}_{0}^{n}$ by

$$
\varphi_{0}(z)=\int_{\gamma(\bar{z}, z)} p d x
$$

where the integral is calculated along an arbitrary path leading from $\bar{z}$ to $z$. This defines $\varphi_{0}$ as a function $\mathbb{V}_{0}^{n} \longrightarrow \mathbb{R}$ such that $d \varphi_{0}(z)=p d x$ if $z=(x, p)\left(\mathbb{V}_{0}^{n}\right.$ being Lagrangian, the integral only depends on the homotopy class with fixed endpoints of $\gamma(\bar{z}, z)$ and since $\mathbb{V}_{0}^{n}$ is in addition simply connected there is just one homotopy class). Let now $H$ be some arbitrary Hamiltonian function; its flow $\left(f_{t}\right)$ takes $\mathbb{V}_{0}^{n}$ to a new Lagrangian manifold $\mathbb{V}_{t}^{n}$ after time $t$. The phase of that manifold is (with obvious notations)

$$
\varphi(z, t)=\varphi_{0}\left(f_{-t}(z)\right)+\int_{\Gamma} p d x-H d t
$$

where the integral is calculated along the arc of phase space trajectory leading from the point $f_{-t}(z) \in \mathbb{V}_{0}^{n}$ at time 0 to the point $z \in \mathbb{V}_{t}^{n}$ at time $t$. Choose now for $H=H_{z_{0}}$; a straightforward calculation (see [5], Prop. 10) yields

$$
\varphi(z, t)=\varphi_{0}\left(z-z_{0}\right)+t p_{0} x-\frac{t^{2}}{2} p_{0} x_{0} .
$$

We next consider, as is customary in geometric quantization (see 2, 3, 4]), a "waveform" on $\mathbb{V}_{0}^{n}$, i.e. an expression of the type

$$
\Psi_{0}(z)=e^{\frac{i}{\hbar} \varphi_{0}(z)} i^{m_{0}(z)} \sqrt{\rho}(z)
$$

where $m_{0}(z)$ is an integer related to the Maslov index and $\rho$ a function $\geq 0$ on $\mathbb{V}_{0}^{n}$ (more accurately, one should view $\sqrt{\rho}$ as a "half-density" on $\mathbb{V}_{0}^{n}$ but we can ignore this point here because half-densities and functions transform the same way under translations). The action of a Hamiltonian flow $\left(f_{t}\right)$ on $\Psi_{0}$ is given by $f_{t} \Psi_{0}(z)=\Psi(z, t)$ where

$$
\Psi(z, t)=e^{\frac{i}{\hbar} \varphi(z, t)} i^{m(z, t)}\left(f_{t}\right)_{*} \sqrt{\rho}(z)
$$

the difference $m(z, t)-m_{0}(z)$ is a count of the number of caustic points appearing when the flow deforms $\mathbb{V}_{0}^{n}$ into $\mathbb{V}_{t}^{n}=f_{t}\left(\mathbb{V}_{0}^{n}\right)$. If $H=H_{z_{0}}$ the manifold $\mathbb{V}_{0}^{n}$ is translated in phase space without any "bending" so that there will appear no new caustics hence $m(z, t)=m_{0}(z)$ and we thus have

$$
\Psi(z, t)=\hat{T}\left(t z_{0}\right) \Psi_{0}(z)
$$

where the operator $\hat{T}\left(z_{0}\right)$ is now defined by

$$
\hat{T}\left(z_{0}\right) \Psi_{0}(z)=e^{\frac{i}{\hbar}\left(p_{0} x-\frac{1}{2} p_{0} x_{0}\right)} T\left(z_{0}\right) \Psi_{0}(z) .
$$


A straightforward calculation shows that the partial differential equation satisfied by $\Psi(z, t)$ is

$$
i \hbar \frac{\partial \Psi}{\partial t}(z, t)=\widehat{\Sigma} \Psi(z, t), \Psi(t=0)=\Psi_{0}
$$

where $\widehat{\Sigma}$ is the operator $\widehat{\Sigma}=-p_{0} x+i \hbar z_{0} \partial_{z}$; the latter can be rewritten in terms of the symplectic form $\sigma$ as

$$
\widehat{\Sigma}=\sigma\left(x_{0}, p_{0} ; x+i \hbar \partial_{p},-i \hbar \partial_{x}\right)
$$

so that (6) is just TF's phase-space Schrödinger equation (10) for the Hamiltonian $H_{z_{0}}$.

The whole argument can actually be reversed: using for instance the methods of characteristics one checks that the solution of equation (6) is precisely (44) so that we conclude that the imposition of the TF quantization rules (2) is equivalent to the extension (15) of Heisenberg-Weyl operators to phase-space.

\section{Weyl Calculus on Phase Space}

The process called "Weyl quantization" associates to a suitable function (or "symbol") $a=a(z)$ the operator $a^{w}=\hat{A}$ defined by

$$
\hat{A} \psi(x)=\left(\frac{1}{2 \pi \hbar}\right)^{n} \int \tilde{a}\left(z_{0}\right) \hat{T}\left(z_{0}\right) \psi(x) d^{2 n} z_{0}
$$

where $\tilde{a}$ is the "alternative Weyl symbol", that is the symplectic Fourier transform $\mathcal{F}_{\sigma} a$ of $a$ :

$$
\tilde{a}(z)=\mathcal{F}_{\sigma} a(z)=\left(\frac{1}{2 \pi \hbar}\right)^{n} \int e^{-\frac{i}{\hbar} \sigma\left(z, z^{\prime}\right)} a\left(z^{\prime}\right) d^{2 n} z^{\prime} .
$$

The discussion of Section 2 suggests that we might now be able to make $\hat{A}$ to act, not only on function of $x$, but also on functions of $z$ by defining

$$
\hat{A} \Psi(z)=\left(\frac{1}{2 \pi \hbar}\right)^{n} \int \tilde{a}\left(z_{0}\right) \widehat{T}\left(z_{0}\right) \Psi(z) d^{2 n} z_{0}
$$

where $\Psi$ now is a function of $z=(x, p)$ and $\widehat{T}\left(z_{0}\right) \Psi(z)$ is given by (5); this expression makes perfectly sense provided of course, that $a$ and $\Psi$ belong to some adequate spaces; we will assume this is the case. It turns out that this reinterpretation of Weyl operators again leads to the TF quantization rules (2). It is sufficient to prove this in the case of one degree of freedom; dropping indices let $\widehat{X}$ and $\widehat{P}$ be the Weyl operators with symbols $x$ and $p$; a simple calculation of (symplectic) Fourier transforms yields

$$
\begin{aligned}
\widehat{X} \Psi(z) & =i \hbar \int \delta\left(x_{0}\right) \otimes \delta^{\prime}\left(p_{0}\right) \widehat{T}\left(z_{0}\right) \Psi(z) d^{2 n} z_{0} \\
& =-i \hbar \int \delta\left(z_{0}\right)\left(\frac{i x}{\hbar} \Psi(z)-\frac{\partial \Psi}{\partial p}(z)\right) d^{2 n} z_{0} \\
& =\left(x+i \hbar \frac{\partial}{\partial p}\right) \Psi(z)
\end{aligned}
$$


and we thus have $\widehat{X}=x+i \hbar \frac{\partial}{\partial p}$; by a similar calculation we get $\widehat{P}=-i \hbar \frac{\partial}{\partial x}$, and we have thus recovered the TF rules (2).

\section{Exact Solutions for Quadratic Hamiltonians}

The extended Weyl calculus constructed in previous Section allows us to solve exactly the TF equation when the Hamiltonian $H$ is a homogeneous quadratic polynomial in the variables $x_{j}, p_{k}$, in which case the Hamiltonian flow consists of symplectic matrices $S_{t}$.

One of the main features (and probably one of the most attractive!) of the usual Weyl correspondence $a \longleftrightarrow a^{w}=\hat{A}$ is the property of metaplectic covariance: if we replace $a$ by $a \circ S^{-1}$ where $S$ is any element of $S p(n)$ then :

$$
\left(a \circ S^{-1}\right)^{w}=\hat{S} a^{w} \hat{S}^{-1}
$$

where $\hat{S}$ is any of the two metaplectic operators $\pm \hat{S}$ associated to $S$ (see [8], Section 6.3; for the properties of $M p(n)$ see [4, Chapter 6). The proof of property (9) is based on the formula

$$
\hat{S} \hat{T}\left(z_{0}\right) \hat{S}^{-1}=\hat{T}\left(S z_{0}\right)
$$

where one uses the traditional definition (3) definition of $\hat{T}\left(z_{0}\right)$, hence if (10) still holds when we redefine $\hat{T}\left(z_{0}\right)$ by (5), then metaplectic covariance (9) will also hold for our enlarged Weyl calculus. Now, Mehlig and Wilkinson have shown 11] that if $\operatorname{det}(S-I) \neq 0$ then

$$
\hat{S}= \pm k \int \hat{T}\left(S z_{0}\right) \hat{T}\left(-z_{0}\right) d^{2 n} z_{0}
$$

where $k$ is a constant given by

$$
k=\left(\frac{1}{2 \pi \hbar}\right)^{n} i^{\nu} \sqrt{|\operatorname{det}(S-I)|}
$$

and $\nu$ is a kind of "Maslov index". We have shown [6] that every $\hat{S} \in M p(n)$ can be written as the product of two operators of the type (11) above (which are only defined for $\operatorname{det}(S-I) \neq 0$ ); we may thus extend every $\hat{S} \in M p(n)$ to an operator defined on functions of $z=(x, p)$; the natural domain of $\hat{S}$ is then $\mathcal{S}\left(\mathbb{R}_{z}^{2 n}\right)$, and can be extended to $L^{2}\left(\mathbb{R}^{2 n}\right)$ and to $\mathcal{S}^{\prime}\left(\mathbb{R}_{z}^{2 n}\right)$ by continuity. Having done this it is immediate to check, using formulae (11) that (10), and hence also (9), remain true in our extended Weyl calculus.

Let us now return to the flow $\left(S_{t}\right)$; denote by $\left(\hat{S}_{t}\right)$ the unique one-parameter group in $M p(n)$ determined by $\left(S_{t}\right)$; viewing the $\hat{S}_{t}$ as extended Weyl operators we automatically have

$$
i \hbar \frac{d}{d t} \hat{S}_{t}=H\left(x+i \hbar \partial_{p},-i \hbar \partial_{x}\right) \hat{S}_{t}
$$

hence $\Psi(z, t)=\hat{S}_{t} \Psi_{0}(z)$ is the solution of the TF equation (1) for the considered Hamiltonian. 


\section{Discussion and Concluding Remarks}

We have established that the Torres-Vega and Frederick's equation fits as part of an extended Weyl calculus, which was itself motivated by considerations from geometric quantization. There remains to find the physical interpretation of that equation. A clue might be the fact that it is possible to rewrite (a variant of) the $\mathrm{TF}$ equation in terms of the star-product, familiar from deformation quantization (this fact seems to have been noticed in [1]; this and the fact that the star-product is used to express the time-evolution of the Wigner function via a quantum-Liouville equation might be an indication that the TF equation plays in quantum mechanics a role similar to that of Hamilton's equation in classical mechanics.

We will come back to this important question in a near future.

\section{References}

[1] D. Chruscinski and K. Mlodawski. Preprint, arXiv: quant-ph/ 0501163 v1, 2005.

[2] M. de Gosson. Bull. Sci. Math. 121:301-322, 1997.

[3] M. de Gosson. Ann. Inst. H. Poincaré, 70(6):547-73, 1999.

[4] M. de Gosson. The Principles of Newtonian and Quantum Mechanics. Imperial College Press, London, 2001.

[5] M. de Gosson. J. Phys. A: Math. Gen. 37(29), 7297-7314, 2004.

[6] M. de Gosson. On the Weyl Representation of Metaplectic Operators. To appear in Lett. Math. Phys., 2005

[7] Jun Lu, Qiam-Shu Li and Zheng-Sun. J. Chem. Phys. 3, 1022-1036, 2001.

[8] R. G. Littlejohn. Physics Reports 138(4-5):193-291, 1986.

[9] Qiam-Shu Li, Jun Lu. Chem. Phys. Letter. 336, 118-122, 2001.

[10] Qiam-Shu Li, Gong Min Wei, and Li Qiang Lu. Phys. Rev. A. 70, 022105 (1-5), 2004.

[11] B. Mehlig and M. Wilkinson. Ann. Phys. 18(10), 6-7, 541-555, 2001.

[12] K. B. Moeller, T. G. Joergensen and G. Torres-Vega. J. Chem. Phys. 106(17), 7228-7240, 1997.

[13] V. Nazaikiinskii, B.-W. Schulze, and B. Sternin. Quantization Methods in Differential Equations. Taylor \& Francis, 2002.

[14] G. Torres-Vega and J. H. Frederick. J. Chem. Phys. 93(12), 8862-8874, 1990. 
[15] G. Torres-Vega and J. H. Frederick. J. Chem. Phys. 98(4), 3103-3120, 1993.

[16] Xu-Guang Hu and Qiam-Shu Li. J. Phys. A: Math. Gen. 32, 139-146, 1999. 